\title{
Enterovesical fistula caused by regressive change of non-Hodgkin's lymphoma: A case report
}

\author{
YU-TING LEE ${ }^{1,2}$, YING-YUAN CHEN ${ }^{2,3}$, CHIA-YUN WU ${ }^{1,2}$, \\ HUNG-MING CHEN ${ }^{4}$, CHENG-HWAI TZENG ${ }^{1,2}$ and TZEON-JYE CHIOU ${ }^{2,5}$
}

\author{
${ }^{1}$ Division of Hematology and Oncology, Department of Medicine, Taipei Veterans General Hospital; \\ ${ }^{2}$ Faculty of Medicine, National Yang-Ming University School of Medicine; ${ }^{3}$ Department of Radiology, \\ Taipei Veterans General Hospital, Taipei 11217; ${ }^{4}$ Division of Hematology and Oncology, Department of Medicine, \\ Taoyuan Armed Forces General Hospital, Taoyuan 32551; ${ }^{5}$ Division of Transfusion Medicine, \\ Department of Medicine, Taipei Veterans General Hospital, Taipei 11217, Taiwan, R.O.C.
}

Received December 19, 2014; Accepted January 22, 2016

DOI: $10.3892 / \mathrm{ol} .2016 .4561$

\begin{abstract}
Enterovesical fistula (EVF) is a rare complication of diverticulitis, as well as Crohn's disease, intestinal malignancy, radiotherapy and trauma. EVF formation is associated with inflammation of the involved bowel segments. The current study presents the case of a 35-year-old man with non-Hodgkin's lymphoma who developed pneumaturia, fecaluria and recurrent urinary tract infections following chemotherapy, accompanied by regressive change of the lymphoma. Abdominal computed tomography scans revealed that the terminal ileum had adhered to the bladder wall. The patient underwent exploratory laparotomy and partial resection of the terminal ileum, and EVF was confirmed. Histological examination revealed an inflammatory response but no evidence of residual lymphoma. The diagnosis of EVF is occasionally difficult and requires appropriate radiographic examination. Surgical treatment is recommended.
\end{abstract}

\section{Introduction}

An enterovesical fistula (EVF) is defined as an abnormal communication between the intestine and the bladder (1). Enterovesical fistula (EVF) may occur as a rare complication of diverticulitis, as well as Crohn's disease, intestinal malignancy, radiotherapy and traumatic or iatrogenic injuries. Patients with EVF most commonly present with lower urinary tract symptoms, including pneumaturia, fecaluria, frequency, urgency, suprapubic pain, recurrent urinary tract infections

Correspondence to: Dr Tzeon-Jye Chiou, Division of Transfusion Medicine, Department of Medicine, Taipei Veterans General Hospital, 201 Shipai Road, Section 2, Taipei 11217, Taiwan, R.O.C.

E-mail: tjchiou@vghtpe.gov.tw

Key words: enterovesical fistula, ileovesical fistula, non-Hodgkin's lymphoma, complication of chemotherapy, intestinal fistula, urinary fistula and hematuria. EVF is a significant morbidity and the associated lower urinary tract symptoms may deteriorate a patient's quality of life (1). The standard treatment for EVF is bowel resection with primary anastomosis, and disease recurrence is rare (1). EVF caused by primary small intestinal non-Hodgkin's lymphoma (NHL) has been previously reported (2), however, EVF developing after cytotoxic therapy or regressive change of lymphoma is extremely rare. NHL had been classified as an indolent and aggressive type of lymphoma. The current study presents the case of a patient with NHL who developed EVF after receiving cytotoxic therapy accompanied by regressive change of the lymphoma. The study was approved by the ethics committee of Taipei Veterans General Hospital, Taipei, Taiwan.

\section{Case report}

A cutaneous marginal zone B-cell lymphoma was identified in the left orbital area in a 35-year-old Taiwanese man during local excision at the Taipei Veterans General Hospital (Taipei, Taiwan) in October 2012. Multiple neck lymphadenopathy with B symptoms developed from January 2014. Excisional biopsy of the neck lymph node was performed. Lymph node tissue $(4-\mu \mathrm{m})$ sections were formalin-fixed, paraffin embedded (Thermo Fisher Scientific, Inc., Waltham, MA, USA) and stained with hematoxylin and eosin (Sigma-Aldrich, St. Louis, MO, USA). Immunohistochemical staining revealed positivity for CD20 (mouse anti-human monoclonal antibody; clone, L-26; 1:300; cat. no. M0755; Dako, Glostrup, Denmark), B-cell lymphoma-6 (mouse anti-human monoclonal antibody; clone, LN22; 1:200; cat. no. NCL-L-BCL-6-564; Leica Microsystems Ltd., Milton Keynes, UK) and MUM1 (mouse anti-human monoclonal antibody; clone, EAU32; 1:1,000; cat. no. NCL-L-MUM1; Leica Microsystems Ltd.). Subsequently, diffuse large B cell lymphoma, a type of non-Hodgkin's lymphoma (NHL), was diagnosed. The clinical stage was IV (3) with involvement of the liver and right kidney, as well as lymphadenopathy of the bilateral neck, terminal ileum and gastrohepatic ligament. Standard chemotherapy with R-CHOP every 3 weeks for 6 cycles, comprising rituximab $\left(375 \mathrm{mg} / \mathrm{m}^{2}\right)$, 

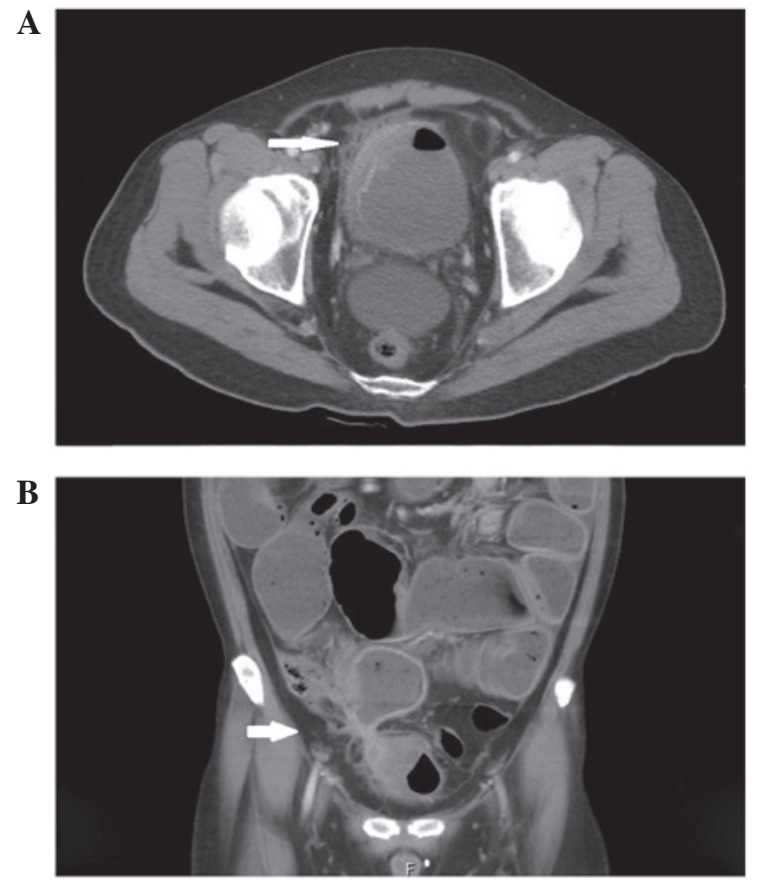

Figure 1. Computed tomography (CT) scans. (A) Abdominal CT revealed an irregular surface and thickening of the bladder wall (arrow), and air in the bladder. (B) Adherence and communication between the terminal ileum and bladder was also observed (arrow).

cyclophosphamide $\left(750 \mathrm{mg} / \mathrm{m}^{2}\right)$, doxorubicin $\left(50 \mathrm{mg} / \mathrm{m}^{2}\right)$, vincristine $\left(1.4 \mathrm{mg} / \mathrm{m}^{2}\right)$ and prednisolone $(100 \mathrm{mg} / \mathrm{day}$ for 5 days), commenced in March 2014.

Although the neck lymphadenopathy exhibited marked regression after the first course of chemotherapy, intermittent nausea and vomiting, abdominal bloating and perineal pain with dysuria developed after the second course of R-CHOP. Laboratory examinations revealed the following results: White blood cell count, 29,000/ $\mu \mathrm{l}$ (normal range, 4,500-11,0000/ $\mu 1$ ); hemoglobin level, $13.7 \mathrm{~g} / \mathrm{dl}$ (normal range, $14-18 \mathrm{~g} / \mathrm{dl}$ ); platelet count, $624 \times 10^{3} / \mu 1$ (normal range, $150-350 \times 10^{3} / \mu 1$ ); blood urea nitrogen level, $4.0 \mathrm{mg} / \mathrm{dl}$ (normal range, $7.0-20.0 \mathrm{mg} / \mathrm{dl}$ ); creatinine level, $1.04 \mathrm{mg} / \mathrm{dl}$ (normal range, $0.7-1.5 \mathrm{mg} / \mathrm{dl}$ ); uric acid level, $3.3 \mathrm{mg} / \mathrm{dl}$ (normal range, $2.5-7.2 \mathrm{mg} / \mathrm{dl}$ ); C-reactive protein level, $4.26 \mathrm{mg} / \mathrm{dl}$ (normal range, $<0.5 \mathrm{mg} / \mathrm{dl}$ ); and lactate dehydrogenase level, $166 \mathrm{U} / \mathrm{ml}$ (normal range, 149-519 U/ml). In the urinary microscopic examination (Eclipse 50i microscope; Nikon Corporation, Tokyo, Japan), >100 red blood cells and $>100$ white blood cells per high-power field (magnification, $\mathrm{x} 40$ ) were identified (normal range, $0-2$ per high-power field).

Empiric antibiotic treatment with intravenous cefuroxime $(1.5 \mathrm{~g}$, every $8 \mathrm{~h})$ was administered to treat the urinary tract infection, however, it was unsuccessful. The patient continued to experience hematuria, pyuria and perineal tenderness. In addition, pneumaturia and fecaluria occurred during hospitalization at the Taipei Veterans General Hospital. A cystoscopic examination revealed an irregular surface, edema and erythematous changes of the bladder wall. An intravenous pyelogram demonstrated no genitourinary structural abnormalities. Urine cytology for malignancy was negative. An acid-fast stain and tuberculosis culture were also negative. Abdominal computed tomography $(\mathrm{CT})$ revealed adherence and communication between the terminal ileum and bladder, with intestinal obstruction and focal wall thickening of the urinary bladder and adjacent intestine (Fig. 1). Furthermore, retrograde cystourethrography was performed; however, this examination also revealed no remarkable findings, other than inflammatory changes. Laparotomy was performed in June 2014, and a fistula between the terminal ileum and bladder was identified. A partial resection of the terminal ileum, as well as partial cystectomy and ileostomy were performed. The resected small intestine and colon, measuring $15 \times 5 \mathrm{~cm}$, contained multiple ulcers and one perforation. The microscopic findings (magnification, $\mathrm{x} 40$; Eclipse 50i microscope; Nikon Corporation) indicated heavy acute and chronic inflammatory cells that infiltrated the whole layer of intestinal wall, but no evidence of lymphoma cells.

The patient experienced a good recovery, without complication. The patient completed 6 cycles of chemotherapy with R-CHOP, and then underwent surgery to close the ileostomy. No recurrence of EVF was indicated during the 22-month follow-up period.

\section{Discussion}

EVF is an abnormal communication between the intestine and the bladder. Depending on the area of the bowel involved, EVF is classified as colovesical, rectovesical, ileovesical or appendicovesical fistulae. The prevalence is $\sim 1$ in every 3,000 surgical hospital admissions (4). The symptoms of EVF include pneumaturia, fecaluria, frequency, urgency, suprapubic pain, recurrent urinary tract infections and hematuria (1).

Inflammatory changes of the bowel wall with adherence to the bladder wall are considered to result in EVF formation (1). EVF is not only a complication of primary small intestine lymphoma but also occurs in patients undergoing treatment of lymphoma. In the current patient, lymphoma with terminal ileum involvement was noted initially. However, it was subsequently identified that a large number of inflammatory cells had infiltrated the bowel wall following chemotherapy. The regressive change of lymphoma cells may have resulted in focal intestinal inflammation and adherence, and led to fistula and intestinal obstruction. Another potential mechanism is a cytotoxic agent associated with regional enteritis following EVF formation (5).

The diagnosis of EVF may be challenging for clinicians and radiologists. Various diagnostic tools and procedures are used to detect EVF. The Taipei Veterans General Hospital prefers to use $\mathrm{CT}$ and magnetic resonance imaging techniques to identify the fistula tract, as they provide a higher detection rate compared with endoscopy or radiographic examination. The accuracy of these diagnostic tests may be associated with the location and characteristics of EVF $(1,6)$. For example, cystourethrography was arranged for the present patient, however, no contrast outside the bladder was shown. When the location of EVF is higher than the contrast agent can reach, contrast cannot be used to track the fistula.

Management of EVF is dependent on the patient's status. As spontaneous closure of the fistula is typically rare, surgical resection is preferred for the majority patients with EVF $(1,6)$. The outcome of surgical resection is good and recurrence is uncommon for patients with benign disease or nonradiation-induced fistulae $(1,6)$, as well as primary 
intestinal NHL (2). However, patients with NHL may have to decide between surgical repair and postponing the scheduled chemotherapy, or delaying the surgery and risk developing sepsis. Thus far, the optimal timing of surgical treatment has not been established. In the present case, surgical repair was arranged and the scheduled chemotherapy was postponed, as the patient was aggravated by the symptoms and the lymphoma was under control. Ansari et al also reported a patient with NHL who developed EVF following chemotherapy and and had an uneventful recovery following a surgical resection (5).

In conclusion, the appearance of EVF is a rare complication, but may develop in patients following cytotoxic treatment. When suspicious of EVF formation, appropriate diagnostic investigation should be used to aid clinicians in the early recognition of EVF and avoid any delay in treatment. Surgical resection of the fistula is feasible.

\section{References}

1. Golabek T, Szymanska A, Szopinski T, Bukowczan J, Furmanek M, Powroznik J and Chlosta P: Enterovesical fistulae: Aetiology, imaging, and management. Gastroenterol Res Pract 2013: 617967, 2013 .
2. Shinji S, Akimaru K, Tsuchiya Y, Shimizu T, Kawamoto M, Iwamoto M, Yamaguchi N, Suzuki H, Yamada T, Nikaido T and Uchida E: Enterovesical fistula caused by non-Hodgkin's lymphoma of the ileum: Report of a case. Surg Today 42: 1005-1009, 2012

3. Cheson BD, Fisher RI, Barrington SF, Cavalli F, Schwartz LH, Zucca E and Lister TA; Alliance, Australasian Leukaemia and Lymphoma Group; Eastern Cooperative Oncology Group; European Mantle Cell Lymphoma Consortium; Italian Lymphoma Foundation; European Organisation for Research; Treatment of Cancer/Dutch Hemato-Oncology Group; Grupo Español de Médula Ósea; German High-Grade Lymphoma Study Group; German Hodgkin's Study Group; Japanese Lymphorra Study Group; Lymphoma Study Association; NCIC Clinical Trials Group; Nordic Lymphoma Study Group; Southwest Oncology Group; United Kingdom National Cancer Research Institute: Recommendations for initial evaluation, staging, and response assessment of Hodgkin and non-Hodgkin lymphoma: The Lugano classification. J Clin Oncol 32: 3059-3068, 2014.

4. Pugh JI: On the pathology and behaviour of acquired non-traumatic vesico-intestinal fistula. Br J Surg 51: 644-657, 1964.

5. Ansari MS, Nabi G, Singh I, Hemal AK and Pandey G: Colovesical fistula an unusual complication of cytotoxic therapy in a case of non-Hodgkin's lymphoma. Int Urol Nephrol 33: 373-374, 2001

6. Scozzari G, Arezzo A and Morino M: Enterovesical fistulas: Diagnosis and management. Tech Coloproctol 14: 293-300, 2010 . 